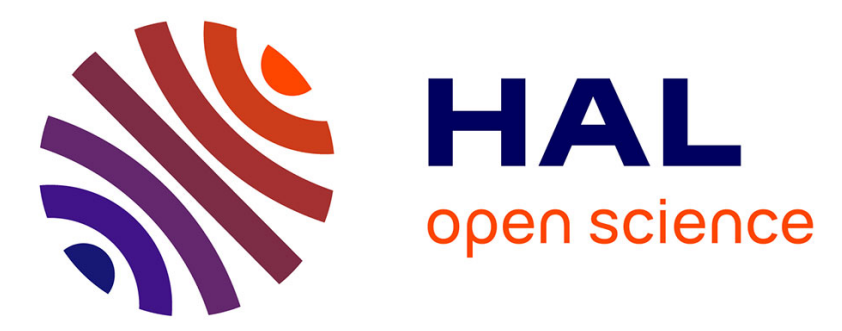

\title{
On simultaneous parameter identification and state estimation for cascade state affine systems
}

\author{
Malek Ghanes, Gang Zheng, Jesus de Leon-Morales
}

\section{To cite this version:}

Malek Ghanes, Gang Zheng, Jesus de Leon-Morales. On simultaneous parameter identification and state estimation for cascade state affine systems. IEEE American Control Confrerence, Jun 2008, Seattle, United States. inria-00531057

\section{HAL Id: inria-00531057 https://hal.inria.fr/inria-00531057}

Submitted on 1 Nov 2010

HAL is a multi-disciplinary open access archive for the deposit and dissemination of scientific research documents, whether they are published or not. The documents may come from teaching and research institutions in France or abroad, or from public or private research centers.
L'archive ouverte pluridisciplinaire HAL, est destinée au dépôt et à la diffusion de documents scientifiques de niveau recherche, publiés ou non, émanant des établissements d'enseignement et de recherche français ou étrangers, des laboratoires publics ou privés. 


\title{
On simultaneous parameter identification and state estimation for cascade state affine systems
}

\author{
M. GHANES, G. ZHENG and J. DE LEON-MORALES
}

\begin{abstract}
In this paper, an adaptive observer is proposed to solve the problem of simultaneous parameter identification and state estimation for a class of cascade state affine systems. Sufficient conditions are given in order to guarantee the exponential convergence of the proposed observer. Furthermore, simulation results are given illustrating the performance of the proposed observer when it is applied in the synchronization and identification problem of Rossler's chaotic system.
\end{abstract}

Index Terms-Adaptive observer, synchronization, identification, Rossler's chaotic system

\section{INTRODUCTION}

Nonlinear observer design is an important problem in the theory of systems. It is clear that no general procedure exists to construct a nonlinear observer for a general nonlinear system. However, for a class of nonlinear systems, under some extra assumptions, such as Lipchitz, persistent exciting..., it is possible to construct the observer. For the purpose of state estimation, many works have been devoted [14],[13],[9],[11]. However, when dealing with the problem of parameter identification, it becomes more difficult. The simultaneous parameter identification and state estimation problem has been attracted the attention of various research groups, since it is very useful to treat many practical problems, such as fault detection, signal transmission or control, and recently for synchronization of chaotic systems. Motivated by this interest, several approaches have been proposed to simultaneously estimate the state and identify the parameters. In [8], author has proposed a novel adaptive observer with an appropriate adaptation law for the unknown parameters. And in [10], the unknown parameters were treated as the extended state of the system, in such a way the existed 'classical' nonlinear observer design methods can be applied to the augmented system. All these methods can be used to design nonlinear observer for a large class of nonlinear system, such as linear time invariant/variant systems, and a class of state affine systems.

In this paper, our goal is firstly to design an adaptive observer in order to estimate the unmeasurable state variables of the system and to identify the unknown parameters simultaneously for a class of cascade state affine systems.

M. GHANES is with ECS/ENSEA, 6 Avenue du Ponceau, 95014 CergyPontoise, France.

G. Zheng is with INRIA Rhône-Alpes, Inovallée, 655 avenue de l'Europe, Montbonnot Saint Martin, 38334 St. Ismier Cedex, France.

J. DE LEON-MORALES is with Universidad Autónoma de Nuevo Leon, Apdo. Postal 148-F, San Nicolas de Los Garza; N. L 66451, Mexico.
Furthermore, some sufficient conditions in order to guarantee the exponential convergence of the proposed adaptive observer are given.

Besides, according to [2], the problem of synchronization of chaotic systems has been related to the concept of observer design from theoretical point of view. Therefore, in order to highlight the feasibility of the proposed observer, we apply it to treat the problem of chaotic synchronization. In fact, the topic of synchronization of chaotic system has attracted many researchers' attention since the work of [3]. After that, many applications of chaotic synchronization have been developed, such as chaotic secure communication based on synchronization of chaotic system [4], [6], [7], [5] and [12].

Inspired by these works, in this paper, secondly, we also try to deal with the synchronization problem for Rossler's chaotic system based on the proposed observer in order to not only estimate the state of system, but also identify its unknown parameters at the same time.

The paper is organized as follows: In section 2, some basic notations about the considered nonlinear systems are introduced. And section 3 is devoted to adaptive observer design for such class of cascade state affine systems. In section 4, an illustrative example dealing with the synchronization and identification parameter problem of Rossler's chaotic systems is given. Simulation results are presented in order to emphasize the performance of the proposed adaptive observer.

\section{NOTATIONS}

In this paper, we are interested in designing an exponential observer for the following cascade state affine system:

$$
\left\{\begin{array}{l}
\dot{z}=A(y, u, z, \theta) z+\beta(y, u, z, \theta)+\varphi(y, u, z, \theta) \theta \\
y=C z
\end{array}\right.
$$

where $z \in R^{n}, u \in R^{l}, y \in R^{p}, \theta \in R^{q}$ are respectively the state, known input, output of the system and the parameter, function $A, \beta, \varphi$ and $C$ are the matrices of appropriate dimensions, and the components of matrix $A$, and vectors $\beta$ and $\varphi$ are continuous functions depending on $\mathrm{u}, \mathrm{y}$ and $z_{1}, \ldots, z_{i-1}, \theta_{1}, \ldots, \theta_{i-1}$, for $1 \leq i \leq p$ and uniformly bounded, with

$$
z=\left(\begin{array}{c}
z_{1} \\
\vdots \\
z_{p}
\end{array}\right), \quad y=\left(\begin{array}{c}
y_{1} \\
\vdots \\
y_{p}
\end{array}\right), \quad \theta=\left(\begin{array}{c}
\theta_{1} \\
\vdots \\
\theta_{p}
\end{array}\right)
$$




$$
\begin{aligned}
& A(y, u, z, \theta) \\
& = \\
& \operatorname{diag}\left(\begin{array}{c}
A_{1}(y, u), \\
\cdots, \\
A_{p}\left(y, u, z_{1}, \ldots, z_{p-1}, \theta_{1}, \ldots, \theta_{p-1}\right)
\end{array}\right), \\
& C=\operatorname{diag}\left(C_{1}, \cdots, C_{p}\right), \\
& \beta(y, u)=\left(\begin{array}{c}
\beta_{1}(y, u) \\
\vdots \\
\beta_{p}\left(y, u, z_{1}, \ldots, z_{p-1}, \theta_{1}, \ldots, \theta_{p-1}\right)
\end{array}\right) \\
& =\begin{array}{c}
\varphi(y, u, z, \theta) \\
\operatorname{diag}\left(\begin{array}{c}
\varphi_{1}(y, u), \\
\cdots, \\
\varphi_{p}\left(y, u, z_{1}, \ldots, z_{p-1}, \theta_{1}, \ldots, \theta_{p-1}\right)
\end{array}\right)
\end{array}
\end{aligned}
$$

where $z_{i} \in R^{n_{i}}, \theta_{i} \in R^{q_{i}}, y_{i} \in R, A_{i}, \beta_{i}, \varphi_{i}, C_{i}$ are the matrices of appropriate dimensions, for $1 \leq i \leq p, u \in R^{l}$. And $\sum_{i=1}^{p} n_{i}=n, \sum_{i=1}^{p} q_{i}=q$.

In the sequel, the dependence of $u, y, z_{1}, \ldots, z_{i-1}, \theta_{1}, \ldots, \theta_{i-1} \quad$ and $\hat{z}_{i-1}, \hat{\theta}_{1}, \ldots, \hat{\theta}_{i-1}$, with $A_{i}, \varphi_{i}, \beta_{i}$ and $\hat{A}_{i}, \hat{\varphi}_{i}, \hat{\beta}_{i}$ will be omitted in order to lighten notations.

\section{AdAPTIVE OBSERVER FOR CASCADE SYSTEMS}

Before introducing our main result, we establish the following assumptions:

Assumption A1. If the input is persistently exciting, in the sense that there exist $\alpha_{2, i}>\alpha_{1, i}>0, T_{1, i}>0$ and $t_{0} \geq 0$ such that for all initial condition $x_{0}$, the following condition for all $t \geq t_{0}$ is satisfied:

$$
\begin{gathered}
\alpha_{1, i} I \leq \\
\int_{t}^{t+T_{1, i}} \Psi_{\left(u, x_{0}, i\right)}^{T}(s, t) C_{i}^{T} \Sigma_{i}(s) C_{i} \Psi_{\left(u, x_{0}, i\right)}(s, t) d s \\
\leq \alpha_{2, i} I
\end{gathered}
$$

where $\Psi_{\left(u, x_{0}, i\right)}$ denotes the transition matrix for the system

$$
\left\{\begin{array}{l}
\dot{z}_{i}=A_{i}\left(y, u, z_{1}, \ldots, z_{i-1}, \theta_{i}, \ldots, \theta_{i-1}\right) z_{i}, \\
y_{i}=C_{i} z_{i}
\end{array}\right.
$$

and $\Sigma_{i}$ is some positive definite bounded matrix, for $1 \leq i \leq p$.

\section{Assumption A2. Considering matrix}

$$
\Lambda=\operatorname{diag}\left\{\Lambda_{1}, \ldots, \Lambda_{p}\right\},
$$

where $\Lambda_{i}$ is a matrix defined by

$$
\dot{\Lambda}_{i}=\left\{A_{i}-S_{i}^{-1} C_{i}^{T} \Sigma_{i} C_{i}\right\} \Lambda_{i}+\varphi_{i}
$$

Assume that $\varphi_{i}$ is persistency exciting so that there exist $\gamma_{2, i}>\gamma_{1, i}>0, T_{2, i}>0$ and $t_{0} \geq 0$ and some positive definite matrix $\Sigma$, such that the following inequality holds

$$
\gamma_{1, i} I \leq \int_{t}^{t+T_{2, i}} \Lambda_{i}^{T}(s) C_{i}^{T} \Sigma_{i}(s) C_{i} \Lambda_{i}(s) d s \leq \gamma_{2, i} I
$$

for all $t \geq t_{0}$ and for $1 \leq i \leq p$.

Assumption A3. We assume that the components of $z$ and $\theta$ are bounded i.e. there exist positive constants such that

$$
\left\|z_{i}\right\| \leq \delta_{i}^{z}, \quad\left\|\theta_{i}\right\| \leq \delta_{i}^{\theta}
$$

and the following inequalities hold

$$
\begin{aligned}
\left\|\hat{A}_{i}-A_{i}\right\| & \leq \sum_{j=1}^{i-1} \delta_{j}^{A}\left\|e_{j}\right\|+\sum_{j=1}^{i-1} \varrho_{j}^{A}\left\|e_{\theta_{j}}\right\| \\
\left\|\hat{\beta}_{i}-\beta_{i}\right\| & \leq \sum_{j=1}^{i-1} \delta_{j}^{\beta}\left\|e_{j}\right\|+\sum_{j=1}^{i-1} \varrho_{j}^{\beta}\left\|e_{\theta_{j}}\right\| \\
\left\|\hat{\varphi}_{i}-\varphi_{i}\right\| & \leq \sum_{j=1}^{i-1} \delta_{j}^{\varphi}\left\|e_{j}\right\|+\sum_{j=1}^{i-1} \varrho_{j}^{\varphi}\left\|e_{\theta_{j}}\right\|
\end{aligned}
$$

where $e_{i}=z_{i}-\hat{z}_{i}$ and $e_{\theta_{i}}=\theta_{i}-\hat{\theta}_{i}$, for $1 \leq i \leq p$.

Theorem 1: Consider system (1), if assumptions A1, A2 and $\mathrm{A} 3$ are satisfied, then the following system

$$
\begin{aligned}
\dot{\hat{z}}= & A(y, u, \hat{z}, \hat{\theta}) \hat{z}+\beta(y, u, \hat{z}, \hat{\theta})+\varphi(y, u, \hat{z}, \hat{\theta}) \hat{\theta} \\
& +\left\{S^{-1} C^{T}+\Lambda \Gamma^{-1} \Lambda^{T} C^{T}\right\} \Sigma(y-C \hat{z})
\end{aligned}
$$

where

$$
\left\{\begin{array}{l}
\dot{\hat{z}}_{i}=\hat{A}_{i} \hat{z}_{i}+\hat{\beta}_{i}+\hat{\varphi}_{i} \hat{\theta}_{i} \\
\quad+\left\{S_{i}^{-1} C_{i}^{T}+\Lambda_{i} \Gamma_{i}^{-1} \Lambda_{i}^{T} C_{i}^{T}\right\} \Sigma_{i}\left(y_{i}-C_{i} \hat{z}_{i}\right) \\
\dot{S}_{i}=-\rho_{i} S_{i}-\hat{A}_{i}^{T} S_{i}-S_{i} \hat{A}_{i}+C_{i}^{T} \Sigma_{i} C_{i} \\
\dot{\Lambda}_{i}=\left\{\hat{A}_{i}-S_{i}^{-1} C_{i}^{T} \Sigma_{i} C_{i}\right\} \Lambda_{i}+\hat{\varphi}_{i} \\
\dot{\Gamma}_{i}=-\lambda_{i} \Gamma_{i}+\Lambda_{i}^{T} C_{i}^{T} \Sigma_{i} C_{i} \Lambda_{i} \\
\dot{\hat{\theta}}_{i}=\Gamma_{i}^{-1} \Lambda_{i}^{T} C_{i}^{T} \Sigma_{i}\left(y_{i}-C_{i} \hat{z}_{i}\right)
\end{array}\right.
$$

is an exponential observer for system (1), where $\rho_{i}$ and $\lambda_{i}$ are sufficiently large positive constants and $\Sigma_{i}$ are some positive definite matrices for $1 \leq i \leq p$.

Proof: Set $e=\left(e_{1}, \ldots, e_{p}\right)^{T}$ and $e_{\theta}=\left(e_{\theta_{1}}, \ldots, e_{\theta_{p}}\right)^{T}$, the state estimation error and the parameter estimation error respectively, where $e_{i}=z_{i}-\hat{z}_{i}$ and $e_{\theta_{i}}=\theta_{i}-\hat{\theta}_{i}$, and $\theta_{i}$ is a constant, for $1 \leq i \leq p$.

For a general case of $i$, for $1 \leq i \leq p$, the estimation error dynamics for $e_{i}=z_{i}-\hat{z}_{i}$ and $e_{\theta_{i}}=\theta_{i}-\hat{\theta}_{i}$ can be obtained as follows

$$
\begin{aligned}
\dot{e}_{i}= & \left(A_{i}-S_{i}^{-1} C_{i}^{T} \Sigma_{i} C_{i}-\Lambda_{i} \Gamma_{i}^{-1} \Lambda_{i}^{T} C_{i}^{T} \Sigma_{i} C_{i}\right) e_{i} \\
& +\varphi_{i} e_{\theta_{i}}+\left(\hat{A}_{i}-A_{i}\right) z_{i}+\left(\hat{\beta}_{i}-\beta_{i}\right) \\
& +\left(\hat{\varphi}_{i}-\varphi_{i}\right) \theta_{i}
\end{aligned}
$$


and

$$
\dot{e}_{\theta_{i}}=-\Gamma_{i}^{-1} \Lambda_{i}^{T} C_{i}^{T} \Sigma_{i} C_{i} e_{i}
$$

Introducing the following change of variable

$$
\epsilon_{i}=e_{i}-\Lambda_{i} e_{\theta_{i}}
$$

we get

$$
\begin{aligned}
\dot{\epsilon}_{i}= & \left(A_{i}-S_{i}^{-1} C_{i}^{T} \Sigma_{i} C_{i}\right) \epsilon_{i}+\left(\hat{A}_{i}-A_{i}\right) z_{i} \\
& +\left(\hat{\beta}_{i}-\beta_{i}\right)+\left(\hat{\varphi}_{i}-\varphi_{i}\right) \theta_{i}
\end{aligned}
$$

Because $S_{i}$ and $\Gamma_{i}$ are some positive definite matrices according to Assumptions A1 and A2, a candidate Lyapunov function can be chosen as follows

$$
v_{i}=\epsilon_{i}^{T} S_{i} \epsilon_{i}+e_{\theta_{i}}^{T} \Gamma_{i} e_{\theta_{i}}
$$

Hence its time derivative is given by

$$
\begin{aligned}
\dot{v}_{i}= & -\rho_{i} \epsilon_{i}^{T} S_{i} \epsilon_{i}-\lambda_{i} e_{\theta_{i}}^{T} \Gamma_{i} e_{\theta_{i}} \\
& -\left(\epsilon_{i}+\Lambda_{i} e_{\theta_{i}}\right)^{T} C_{i}^{T} \Sigma_{i} C_{i}\left(\epsilon_{i}+\Lambda_{i} e_{\theta_{i}}\right) \\
& +2 \epsilon_{i}^{T} S_{i}\left\{\begin{array}{c}
\left(\hat{A}_{i}-A_{i}\right) z_{i}+\left(\hat{\beta}_{i}-\beta_{i}\right) \\
+\left(\hat{\varphi}_{i}-\varphi_{i}\right) \theta_{i}
\end{array}\right\}
\end{aligned}
$$

According to Assumptions A1, A2 and A3, we obtain

$$
\begin{aligned}
\dot{v}_{i} \leq \quad & -\rho_{i} \epsilon_{i}^{T} S_{i} \epsilon_{i}-\lambda_{i} e_{\theta_{i}}^{T} \Gamma_{i} e_{\theta_{i}} \\
+ & +2\left\|\epsilon_{i}\right\|\left\|S_{i}\right\|\left\{\delta_{i}^{z}\left(\sum_{j=1}^{i-1} \delta_{j}^{A}\left\|e_{j}\right\|+\sum_{j=1}^{i-1} \varrho_{j}^{A}\left\|e_{\theta_{j}}\right\|\right)\right\} \\
+ & \left\|\epsilon_{i}\right\|\left\|S_{i}\right\|\left\{\sum_{j=1}^{i-1} \delta_{j}^{\beta}\left\|e_{j}\right\|+\sum_{j=1}^{i-1} \varrho_{j}^{\beta}\left\|e_{\theta_{j}}\right\|\right\} \\
+ & \left\|\epsilon_{i}\right\|\left\|S_{i}\right\|\left\{\delta_{i}^{\theta}\left(\sum_{j=1}^{i-1} \delta_{j}^{\varphi}\left\|e_{j}\right\|+\sum_{j=1}^{i-1} \varrho_{j}^{\varphi}\left\|e_{\theta_{j}}\right\|\right)\right\}
\end{aligned}
$$

which can be rearranged as follows

$$
\begin{aligned}
\dot{v}_{i} \leq & -\rho_{i} \epsilon_{i}^{T} S_{i} \epsilon_{i}-\lambda_{i} e_{\theta_{i}}^{T} \Gamma_{i} e_{\theta_{i}} \\
& +2\left\|\epsilon_{i}\right\|\left\|S_{i}\right\| \sum_{j=1}^{i-1}\left\{\delta_{i}^{z} \delta_{j}^{A}+\delta_{j}^{\beta}+\delta_{i}^{\theta} \delta_{j}^{\varphi}\right\}\left\|e_{j}\right\| \\
& +2\left\|\epsilon_{i}\right\|\left\|S_{i}\right\| \sum_{j=1}^{i-1}\left\{\delta_{i}^{z} \varrho_{j}^{A}+\varrho_{j}^{\beta}+\delta_{i}^{\theta} \varrho_{j}^{\varphi}\right\}\left\|e_{\theta_{j}}\right\|
\end{aligned}
$$

Hence, using the following inequality

$$
\|x\|\|y\| \leq \frac{1}{2}\left(\|x\|^{2}+\|y\|^{2}\right)
$$

we get

$$
\begin{aligned}
\dot{v}_{i} \leq & -\rho_{i} \epsilon_{i}^{T} S_{i} \epsilon_{i}-\lambda_{i} e_{\theta_{i}}^{T} \Gamma_{i} e_{\theta_{i}} \\
& +\sum_{j=1}^{i-1}\left\{L_{j, 1}^{i}+L_{j, 2}^{i}\right\}\left\|\epsilon_{i}\right\|^{2}+\sum_{j=1}^{i-1} L_{j, 1}^{i}\left\|e_{j}\right\|^{2} \\
& +\sum_{j=1}^{i-1} L_{j, 2}^{i}\left\|e_{\theta_{j}}\right\|^{2}
\end{aligned}
$$

where

$$
\begin{aligned}
& L_{j, 1}^{i}=\left\|S_{i}\right\|\left\{\delta_{i}^{z} \delta_{j}^{A}+\delta_{j}^{\beta}+\delta_{i}^{\theta} \delta_{j}^{\varphi}\right\} \\
& L_{j, 2}^{i}=\left\|S_{i}\right\|\left\{\delta_{i}^{z} \varrho_{j}^{A}+\varrho_{j}^{\beta}+\delta_{i}^{\theta} \varrho_{j}^{\varphi}\right\}
\end{aligned}
$$

for $1 \leq i \leq p, 1 \leq j \leq i-1$, with $L_{j, 1}^{1}=L_{j, 1}^{1}=0$.

By applying

$$
\|x+y\|^{2} \leq 2\left(\|x\|^{2}+\|y\|^{2}\right)
$$

and after straightforward computations, we obtain

$$
\begin{aligned}
\dot{v}_{i} \leq & -\left\{\rho_{i}-\frac{\sum_{j=1}^{i-1}\left(L_{j, 1}^{i}+L_{j, 2}^{i}\right)}{\eta_{i}}\right\} \epsilon_{i}^{T} S_{i} \epsilon_{i} \\
& -\lambda_{i} e_{\theta_{i}}^{T} \Gamma_{i} e_{\theta_{i}} \\
& +\sum_{j=1}^{i-1}\left\{\frac{\left.2 L_{j, 1}^{i} \epsilon_{j}^{T} S_{j} \epsilon_{j}\right\}}{\eta_{j}}\right. \\
& +\sum_{j=1}^{i-1}\left\{\frac{2 L_{j, 1}^{i}\left\|\Lambda_{j}\right\|^{2}+L_{j, 2}^{i}}{\chi_{j}} e_{\theta_{j}}^{T} \Gamma_{j} e_{\theta_{j}}\right\}
\end{aligned}
$$

which follows

$$
\dot{v}_{i} \leq-\mu_{i} v_{i}+\sum_{j=1}^{i-1} \kappa_{i, j} v_{j}
$$

where $\mu_{i}=\min \left\{\rho_{i}-\frac{\sum_{j=1}^{i-1}\left(L_{j, 1}^{i}+L_{j, 2}^{i}\right)}{\eta_{i}}, \lambda_{i}\right\} \quad$ and $\kappa_{i, j}=\max \left\{\frac{2 L_{j, 1}^{i}}{\eta_{j}}, \frac{2 L_{j, 1}^{i}\left\|\Lambda_{j}\right\|^{2}+L_{j, 2}^{i}}{\chi_{j}}\right\}$, with $\kappa_{1, j}=0$.

As a result, the whole Lyapunov function can be chosen as $v=\sum_{i=1}^{p} v_{i}$, whose time derivative is $\dot{v}=\sum_{i=1}^{p} \dot{v}_{i}$, and we have

$$
\begin{aligned}
\dot{v} & \leq \sum_{i=1}^{p}\left\{-\mu_{i} v_{i}+\sum_{j=1}^{i-1} \kappa_{i, j} v_{j}\right\} \\
& =\sum_{i=1}^{p}\left(-\mu_{i}+\sum_{j=i+1}^{p} \kappa_{j, i}\right) v_{i}
\end{aligned}
$$

Consequently, if $\mu=\min \left\{\mu_{i} \mid \mu_{i}>\sum_{j=i+1}^{p} \kappa_{j, i}, 1 \leq i \leq p\right\}$, then we obtain

$$
\dot{v} \leq-\mu v<0
$$

and this ends the proof. 


\section{APPLICATION INTO CHAOTIC SYNCHRONIZATION AND PARAMETER IDENTIFICATION}

As an illustrative example of adaptive state affine observation, let us consider the synchronization and parameter identification problem of a chaotic system. It is well-known that a chaotic system is a nonlinear deterministic system having a complex and unpredictable behavior. The sensitive dependence on initial conditions and the parameter variations is a prominent feature of chaotic behavior. In the sequel, we apply the proposed observer to simultaneously estimate states and identify parameters for a given chaotic system.

\section{A. Chaotic system}

Considering the following 3-dimensional autonomous Rossler's chaotic system described by [15]

$$
\left\{\begin{array}{l}
\dot{x}_{1}=-x_{2}-x_{3} \\
\dot{x}_{2}=x_{1}+a x_{2} \\
\dot{x}_{3}=b+x_{3}\left(x_{1}-c\right)
\end{array}\right.
$$

where $x_{i}(1 \leq i \leq 3)$ are the state variables, and $a, b, c$ are all positive real constant parameters. The actual system parameters of (4) are set to $a=0.2, b=0.2$, and $c=5.7$ for exhibiting the chaos phenomenon.

\section{B. Change of coordinate and transformation into cascade system}

By considering the following change of coordinate

$$
\left(\begin{array}{l}
z_{1} \\
z_{2}
\end{array}\right)=\left(\begin{array}{c}
z_{1,1} \\
z_{1,2} \\
z_{2,1}
\end{array}\right)=\left(\begin{array}{c}
x_{1} \\
x_{2} \\
x_{2}+x_{3}
\end{array}\right)
$$

where $z_{1} \in R^{2}, z_{2} \in R$, the chaotic system (4) can be rewritten into cascade system (1) as follows:

$$
\left\{\begin{array}{l}
\dot{z}_{1}=A_{1}(y, u) z_{1}+\beta_{1}(y, u)+\varphi_{1}(y, u) \theta_{1} \\
\dot{z}_{2}=A_{2}\left(y, u, z_{1}, \theta_{1}\right) z_{2}+\beta_{2}\left(y, u, z_{1}, \theta_{1}\right) \\
\quad+\varphi_{2}\left(y, u, z_{1}, \theta_{1}\right) \theta_{2} \\
y_{1}=z_{1,2} \\
y_{2}=z_{2}
\end{array}\right.
$$

where $y_{1} \in R$ and $y_{2} \in R$, with

$$
\left\{\begin{array}{l}
A_{1}(y, u)=\left(\begin{array}{ll}
0 & 0 \\
1 & 0
\end{array}\right), \\
\beta_{1}(y, u)=\left(\begin{array}{c}
-y_{2} \\
0
\end{array}\right), \\
\varphi_{1}(y, u)=\left(\begin{array}{c}
0 \\
y_{1}
\end{array}\right), \\
\theta_{1}=a,
\end{array}\right.
$$

and

$$
\left\{\begin{array}{l}
A_{2}\left(y, u, z_{1}, \theta_{1}\right)=z_{1,1} \\
\beta_{2}\left(y, u, z_{1}, \theta_{1}\right)=z_{1,1}+y_{1}\left(a-z_{1,1}\right) \\
\varphi_{2}\left(y, u, z_{1}, \theta_{1}\right)=\left(\begin{array}{ll}
1 & y_{2}-y_{1}
\end{array}\right) \\
\theta_{2}=\left(\begin{array}{ll}
b & c
\end{array}\right)
\end{array}\right.
$$

From figure 4 , the chaotic system (5) with the parameters $a=0.2, b=0.2, c=5.7$ and initial conditions $z_{1,1}=2$, $z_{1,2}=3, z_{2}=2$, exhibits the chaotic dynamics.

Now, for this cascade system (5), the proposed adaptive observer (2) is applied and designed as follows:

$$
\left\{\begin{aligned}
\dot{z}_{1} & =\hat{A}_{1}(y, u) \hat{z}_{1}+\hat{\beta}_{1}(y, u)+\hat{\varphi}_{1}(y, u) \hat{\theta}_{1} \\
& +\left\{S_{1}^{-1} C_{1}^{T}+\Lambda_{1} \Gamma^{-1} \Lambda_{1}^{T} C_{1}^{T}\right\} \Sigma_{1}\left(y_{1}-C_{1} \hat{z}_{1}\right) \\
\dot{S}_{1} & =-\rho_{1} S_{1}-\hat{A}_{1}^{T} S_{1}-S_{1} \hat{A}_{1}+C_{1}^{T} \Sigma_{1} C_{1} \\
\dot{\Lambda}_{1} & =\left\{\hat{A}_{1}-S_{1}^{-1} C_{1}^{T} \Sigma_{1} C_{1}\right\} \Lambda_{1}+\hat{\varphi}_{1} \\
\dot{\Gamma}_{1} & =-\lambda_{1} \Gamma_{1}+\Lambda_{1}^{T} C_{1}^{T} \Sigma_{1} C_{1} \Lambda_{1} \\
\dot{\hat{\theta}}_{1} & =\Gamma_{1}^{-1} \Lambda_{1}^{T} C_{1}^{T} \Sigma_{1}\left(y_{1}-C_{1} \hat{z}_{1}\right) \\
\dot{\hat{z}}_{2} & =A_{2}\left(y, u, \hat{z}_{1}, \hat{\theta}_{1}\right) \hat{z}_{2}+\hat{\beta}_{2}\left(y, u, \hat{z}_{1}, \hat{\theta}_{1}\right) \\
& +\hat{\varphi}_{2}\left(y, u, \hat{z}_{1}, \hat{\theta}_{1}\right) \hat{\theta}_{2} \\
& +\left\{S_{2}^{-1} C_{2}^{T}+\Lambda_{2} \Gamma^{-1} \Lambda_{2}^{T} C_{2}^{T}\right\} \Sigma_{2}\left(y_{2}-C_{2} \hat{z}_{2}\right) \\
\dot{S}_{2} & =-\rho_{2} S_{2}-\hat{A}_{2}^{T} S_{2}-S_{2} \hat{A}_{2}+C_{2}^{T} \Sigma_{2} C_{2} \\
\dot{\Lambda}_{2} & =\left\{\hat{A}_{2}-S_{2}^{-1} C_{2}^{T} \Sigma_{2} C_{2}\right\} \Lambda_{2}+\hat{\varphi}_{2} \\
\dot{\Gamma}_{2} & =-\lambda_{2} \Gamma_{2}+\Lambda_{2}^{T} C_{2}^{T} \Sigma_{2} C_{2} \Lambda_{2} \\
\dot{\hat{\theta}}_{2} & =\Gamma_{2}^{-1} \Lambda_{2}^{T} C_{2}^{T} \Sigma_{2}\left(y_{2}-C_{2} \hat{z}_{2}\right)
\end{aligned}\right.
$$

where

$$
\left\{\begin{array}{l}
\hat{z}_{1}=\left[\hat{z}_{1,1}, \hat{z}_{1,2}\right]^{T}, \hat{A}_{1}=A_{1}(y, u), \hat{\beta}_{1}=\beta_{1}(y, u) \\
\hat{\varphi}_{1}=\varphi_{1}(y, u), \hat{\theta}_{1}=\hat{a}, C_{1}=\left(\begin{array}{ll}
0 & 1
\end{array}\right)
\end{array}\right.
$$

and

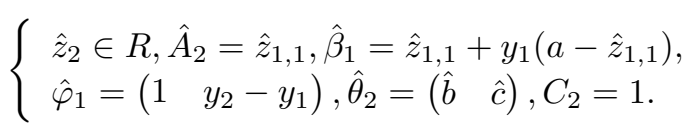

\section{Simulation results}

The aim here is to illustrate the simulation results obtained by the proposed cascade observer when it is applied in the synchronization and parameter identification problem of Rossler's chaotic system. The chosen numerical values (initial conditions and parameters) for Rossler's chaotic cascade system (5) are given in section (IV-B) while the values of its observer (6) are as follows.

The initial conditions are

$\hat{z}_{1,1}=\hat{z}_{1,2}=\hat{z}_{2}=0 ; \hat{\theta}_{1}=\hat{a}=0, \hat{\theta}_{2}=\left(\begin{array}{ll}\hat{b} & \hat{c}\end{array}\right)=0_{1 \times 2}$.
$S_{1}(0)=2 I_{2 \times 2}, \Lambda_{1}(0)=0_{2 \times 2}, \Gamma_{1}(0)=10 I_{2 \times 2} ; S_{2}(0)=1$,
$\Lambda_{2}(0)=0_{1 \times 2}, \Gamma_{2}(0)=10 I_{2 \times 2}$.
The gain were chosen as $\rho_{1}=20, \lambda_{1}=15 ; \rho_{2}=20$, The gain
$\lambda_{2}=15$.

The simulation results obtained with the proposed observer are illustrated in Figs. 1 to 7. 


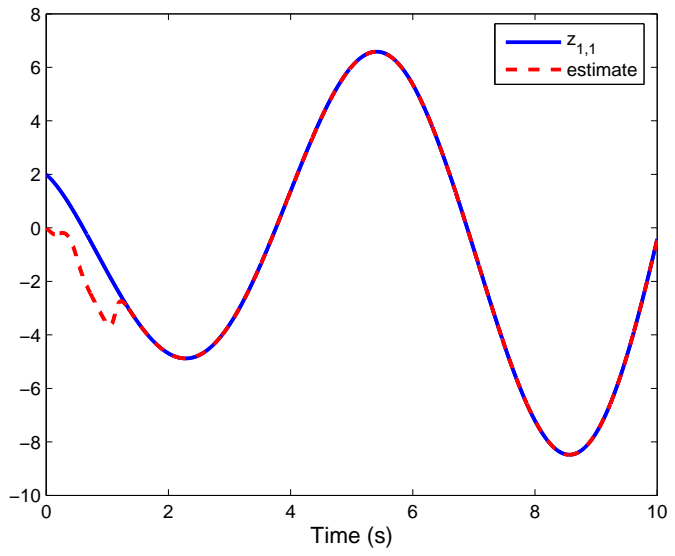

Fig. 1. $z_{1,1}$ and its estimate

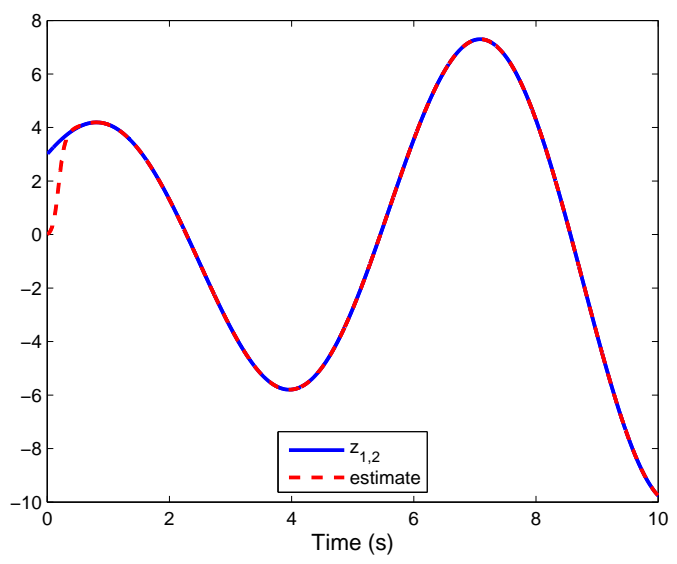

Fig. 2. $z_{1,2}$ and its estimate

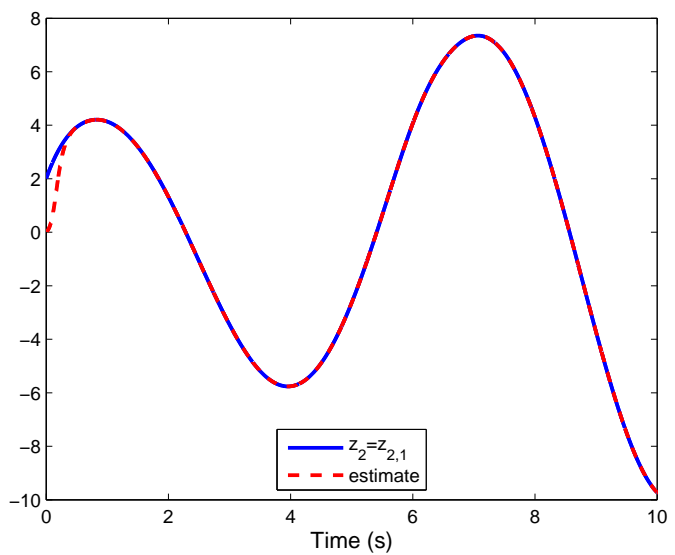

Fig. 3. $z_{2}$ and its estimate

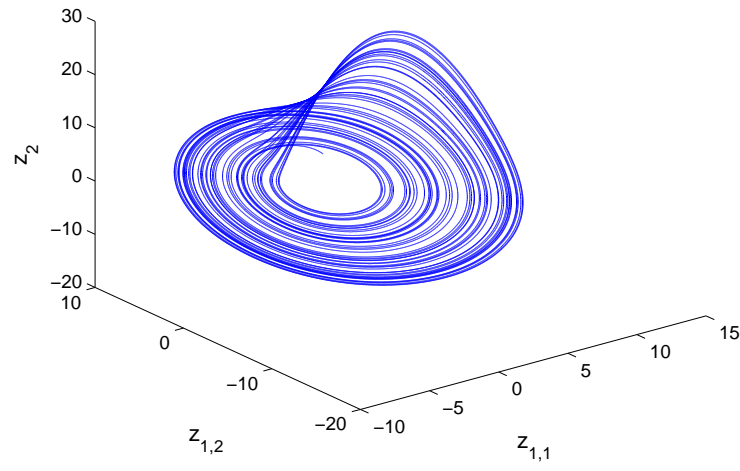

Fig. 4. Three-dimensional phase portrait of $z_{1,1}, z_{1,2}$ and $z_{2}$ with initial conditions $z_{1,1}=2, z_{1,2}=3$, and $z_{2}=2$.

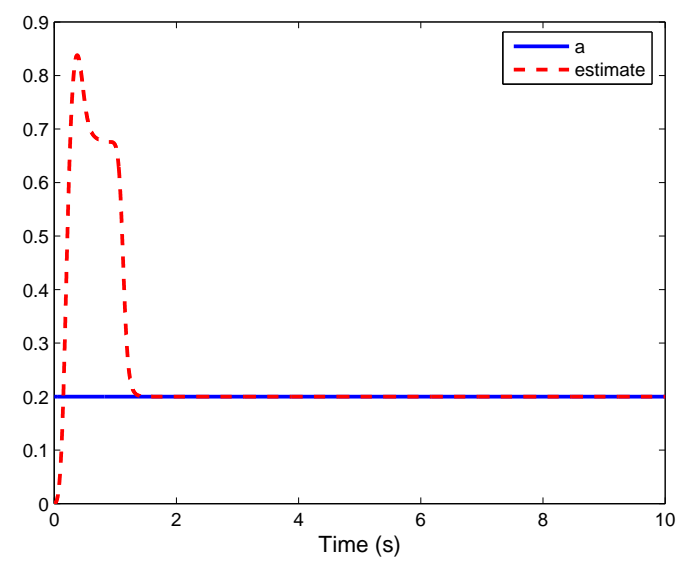

Fig. 5. Parameter $a$ and its estimate

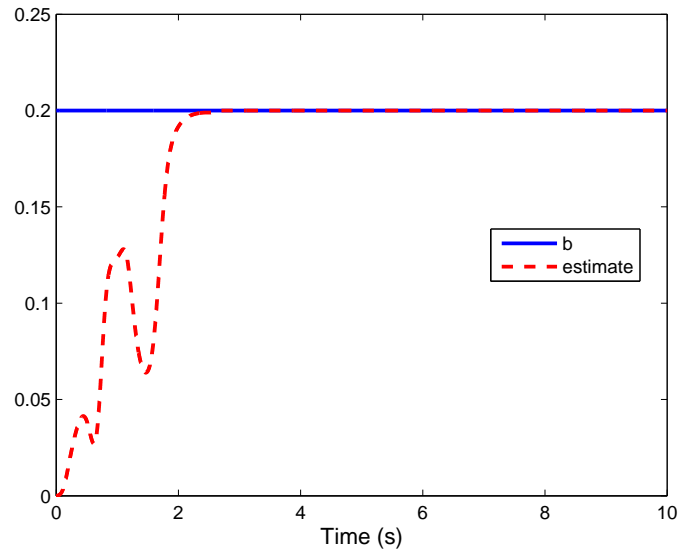

Fig. 6. Parameter $b$ and its estimate 


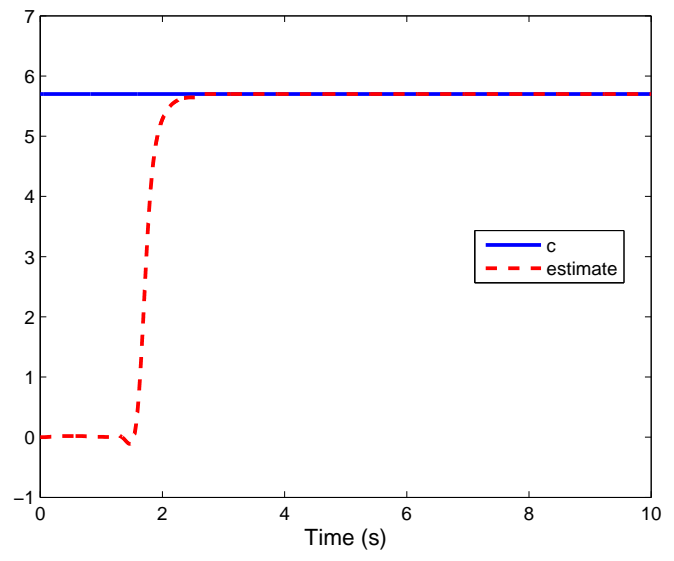

Fig. 7. Parameter $c$ and its estimate

Figs 1, 2 and 3 illustrate the simulation results of the estimation of state variables, i.e. the problem of synchronization. The parameter identification results are depicted from Figs. 5, 6 and 7. Simulation results show that the proposed cascade state affine observer performs well. In all cases, state variables $z_{1,1}, z_{1,2}, z_{2}$ and parameters $a, b, c$ indeed appear to be well estimated.

\section{CONCLUSION}

In this paper, the problem of adaptive observer for a class of cascade state affine systems has been discussed. Sufficient conditions have been given in order to guarantee the exponential convergence of the adaptive observer. Furthermore, it has been shown that the adaptive observer has an arbitrarily tunable rate. One practical interest of such observer is to study the synchronization and parameter identification problem of chaotic systems. An example of synchronization and parameter identification of Rossler's chaotic system has been studied in order to illustrate the feasibility of the proposed observer.

\section{REFERENCES}

[1] Qi G.Y., Du S.Z., Chen G.R. et al. On a four-dimensional chaotic system. Chaos, Solitons and Fractals, 23(2005).

[2] Nijmeijer H. and Mareels I. M. Y. An observer looks at synchronization. IEEE Trans. on Circuits and Systems-1: Fundamental theory and Applications, Vol 44, No 10, pp 882-891, 1997

[3] Pecora L. M. and Carroll T. L. Synchronization in chaotic systems. Physical Review Letters 64, 821-824, 1990.

[4] L. Kovarev, K. S. Eckert, L. O. Chua and U. Parlitz, "Experimental demonstration of secure communications via chaotic synchrionization”, Int. J. Bifurcation and Chaos 2, 709-713, 1992.

[5] Feldmanne U., Hasler M. and Schwarz W. Communication by chaotic signals: The inverse system approach. Int. J. Cricuit Theory and Applications 24. 551-576,1996.

[6] Parlitz U., Chua L. O., Kocarev L. et al. Transmission of digital signals by chaotic synchronization. Int. J. Bifurcation and Chaos 2, 973-977, 1992.

[7] Wu C. W. and Chua L. O. A simple way to synchronize chaotic systems with applications to secure communications systems. Int. J. Bifurcation and Chaos 3, 1619-1627, 1993.

[8] Q. Zhang, Adaptive observers for MIMO linear time-varying systems, IEEE TAC, 47, pp. 525-529, 2002.
[9] H. Hammouri, J. De Leon-Morales, Observers synthesis for state affine systems, in IEEE CDC 1990, pp. 784-785.

[10] G. Besancon, J. De Leon-Morales, et al, On adaptive observers for state affine systems, International journal of control, vol 79, no.6, 2006, pp. 581-591.

[11] G. Besancon, Remarks on nonlinear adaptive observer design, System control letters, 41, pp. 271-280,2000.

[12] U. Parlitz, L.O. Chua, Lj. Kocarev, K. S. Halle and A. Sang, 'Transmission of digital signals by chaotic synchronization', Int. Journal of Bifurcations and Chaos Vol. 2, pp 973-977, 1992.

[13] G. Bastin and M. Gevers, Stable adaptive observers for nonlinear time varying systems, IEEE TAC, vol 33, no 7. 1988, pp:650-658.

[14] G. Kreisselmeier, Adaptive observers with exponential rate of convergence, IEEE TAC 22, pp. 2-8, 1977.

[15] Wei-Der Chang, Parameter identification of Rossler's chaotic system by an evolutionary algorithm, Chaos, Solitons and Fractals, 29, pp. 1047-1053, 2006. 